\title{
Thyroid Function as a Predictive Tool for The Prognosis of Traumatic Patients Admitted in Surgery ICUs
}

Ramin Azhough ${ }^{1}$, Reza Movassaghi ${ }^{*}$, Ali Farbod $^{1}$

1. Department of Surgery, Tabriz University of Medical Sciences, Tabriz, Iran

2. Department of Anesthesiology and Intensive Care Medicine, Tabriz University of Medical Sciences, Tabriz, Iran

\begin{abstract}
Background: APACHE Score is an important criterion for determining the patient prognosis, especially in critically ill patients. According to significantly changes in the serum levels of thyroidal hormones in patients, especially in critically ill patients, the aim of the present study was to evaluate the relationship between thyroid function and APACHE 4 score in the prognosis of the traumatic patients admitted in surgery ICU. Method \& Material: In a descriptiveanalytical study, 90 patients with multiple trauma were studied. Thyroid hormones and APACHE 4 Score were evaluated on 1st, 5th and10th days of admission for each patient. Their clinical features and duration of hospitalization, either in trauma unit or ICU, elation between thyroidal hormones and APACHE 4 Score, duration of hospitalization, and survival of patients were evaluated. Results: This study included 90 patients. A statistically significant relation was detected between thyroid hormones (TT3) and APACHE 4 Score and duration of hospitalization. Conclusion: TT3 might be utilized as a prognostic factor in the traumatic patients admitted to ICU.

Keywords: Trauma; Thyroid; ICU; APACHE;
\end{abstract}

\section{Corresponding author:}

Reza Movassaghi, MD

Department of Anesthesiology and Intensive Care Medicine, Tabriz University of Medical

E-mail: rz_movassaghi@yahoo.com Phone: 09141121090

Receive date: 2017-03-15 | Accept date: 2017-04-28 | Publish date: 2017-05-05

DOI: 10.7575/aiac.abcmed.17.05.03.04 


\section{Introduction}

Determining a complete factor for the prediction of mortality and assessment of the prognosis in ICU patients has many benefits. One of these benefits is utilization of these factors for determining the efficiency in several intensive care units (1). For this purpose, tools such as SAPS (simplified acute physiological system), APACHE (acute physiological and clinical health scoring system), MPM (mortality probability model) and etc. are commonly used. Many studies have been performed to evaluate prognostic power of several predicting factors. One of these studies, conducted by Keegan et al., suggests that the power of mortality prediction in APACHE score 3 And 4 is approximately equal and both of them are better than SAPS which is better than MPM3 (2). Thus, in this study we used APACHE Score 4 as a prognostic determining scoring system.

In sepsis or other clinical illnesses such as trauma, release of tissue stress factors can lead to hypermetabolic state, increasing the consumption of energy, hyperglycemia etc. (34). In sick euthyroid syndrome, the plasma levels of the thyroid hormones, in the absence of thyroidal disease and impaired hypothalamic-hypophysis-thyroid axis, changes (5). The most thyroidal hormone whose plasma level changes during non-thyroidal illnesses diseases is TT-3 (total tri-iodothyronine). TT-3 decreases during these diseases. The most important mechanism proposed for the decrease of TT-3 in non-thyroidal illness is decrease of T4 deiodinization enzymes, and decrease in binding capacity of thyroidal binding proteins (6).

According to high prevalence of the trauma in our country (7), and very few studies that have been conducted for the prediction criteria and power of several scoring systems in traumatic patients, we were to determine relation between thyroidal hormones and
APACHE score for the prognosis of traumatic patients admitted to intensive care units.

\section{Patients and Methods}

In a descriptive-analytical study conducted between March 2015 to June 2016, 90 traumatic patients admitted to ICU were included in this study. Inclusion criteria were traumatic patients admitted to ICU, patients with the age of 18 and older and the lack of history of thyroid illness or surgery. Patients with the history of thyroidal disease or surgery, other metabolic or hormonal diseases which could affect the thyroid function, trauma to the head and neck were excluded from this study. Physiological, clinical, and demographic data were prospectively collected and entered in to a computerized database. 90 traumatic ICU patients were selected and randomized with the rand list version 1.2 software. For each patient TFT (TSH, FT-4, TT-4, TT-3) and APACHE score 4 were measured and detected on the 1st, 5th and10th days of admission. Patients' clinical statuses were followed during the hospitalization. ICU length of stay, hospitalization, and prognosis of each patient were recorded. Statistical analysis was carried out using SPSS15 software. Results were expressed as percentage and mean $\pm S D$. Correlation between variables was assessed with Pearson correlation. P-Value less than 0.05 was considered as the significant level.

\section{Results}

In this study 90 patients were studied, 36 of which (40\%) were female, and 54 patients $(60 \%)$ were male. Ultimately,13 patients (14.4\%) died and 77 patients (85.6\%) survived. The average age of patients was $46.06 \pm 14.21$ years. Average days of hospitalization for dead patients was $21.46 \pm 9.11$ days and for survived patients was $11.37 \pm 5.32$ days and ICU length of stay for dead patients was $15.61 \pm 7.65$ days and for survived patients was $5.48 \pm 4.43$ days. 


\begin{tabular}{|c|c|c|c|c|c|c|}
\hline & \multirow[b]{2}{*}{ Days } & \multicolumn{2}{|c|}{ Alive } & \multicolumn{2}{|c|}{ Dead } & \multirow[b]{2}{*}{$\begin{array}{c}\text { P- } \\
\text { Value }\end{array}$} \\
\hline & & Mean & $\begin{array}{c}\text { Std - } \\
\text { Deviation }\end{array}$ & Mean & $\begin{array}{c}\text { Std- } \\
\text { Deviation }\end{array}$ & \\
\hline & 1 & 2.12 & 1.15 & 2.02 & 1.16 & 0.770 \\
\hline \multirow[t]{3}{*}{ TSH } & 5 & 2.16 & 1.12 & 1.94 & 1.14 & 0.514 \\
\hline & 10 & 1.87 & 0.99 & 1.41 & 0.77 & 0.119 \\
\hline & 1 & 0.85 & 0.16 & 0.83 & 0.13 & 0.351 \\
\hline \multirow[t]{3}{*}{$\mathrm{FT}_{4}$} & 5 & 0.79 & 0.12 & 0.81 & 0.08 & 0.038 \\
\hline & 10 & 0.76 & 0.11 & 0.79 & 0.06 & 0.003 \\
\hline & 1 & 6.79 & 2.16 & 6.27 & 2.73 & 0.446 \\
\hline \multirow[t]{3}{*}{$\mathrm{TT}_{4}$} & 5 & 6.85 & 2.20 & 6.31 & 2.84 & 0.437 \\
\hline & 10 & 6.84 & 2.15 & 6.36 & 2.76 & 0.484 \\
\hline & 1 & 83.54 & 14.62 & 73.53 & 13.30 & 0.023 \\
\hline \multirow[t]{3}{*}{$\mathrm{TT}_{3}$} & 5 & 82.70 & 13.27 & 71.61 & 8.78 & 0.005 \\
\hline & 10 & 82.15 & 12.51 & 70.69 & 11.82 & 0.003 \\
\hline & 1 & 53.6 & 18.7 & 76.6 & 13.5 & 0.000 \\
\hline APACHE & 5 & 44.6 & 17.5 & 70.7 & 14.2 & 0.000 \\
\hline Score & 10 & 39.5 & 17.3 & 69.8 & 20.4 & 0.000 \\
\hline
\end{tabular}

Table 1: Relation between TFT with APACHE score in dead and survived patients

Relation between TFT with APACHE score between dead and survived patients is shown in Table 1. As can be seen, a significant correlation between APACHE score and TT-3 (regardless of the days measured for each patient) and TT-4 (on 5th and 10th days of admission for each patient) was observed.

\section{Discussion}

According to this study, the relation between TT-3 and TT-4 with PACHE score is significant. This study is one of the first studies that have been done for the prognostic value of TFT in traumatic patients. Although, many studies for detection of prognostic value of different hormones have been done, the most important hormone which has been proposed as the best prognostic factor in ill patients is TT3. It has been thought that decrease in the peripheral deiodination of T4 or decrease in binding capacity of thyroidal hormones binding protein, in the serum or tissue, was the major cause of serum level alternations of thyroid hormones during non-thyroidal illness. Thyrotropin serum levels could decrease in acute diseases, so it can cause alternations, specially decrease in the serum levels of T3 and T4, which in turns can lead to ileus, resistance to insulin, increased TG, hyperglycemia etc. (8). Similar to our study, other studies have suggested the serum levels of TT- 3 and TT- 4 to be significantly lower in dead than survived patients. Consequently, serum levels of TT-3 
and $\mathrm{TT}-4$ can be used as a prognostic factor in ICU patients (9).

Numerous studies have been previously performed for detection of any probable relation between hormones and prognosis in the ICU patients. These studies clarify that there is a relationship between hormones and mortality in ICU patients. Wade et al. proposed that cortisol, aldosterone and DHEAS increased in ICU patients and they can be used as prognostic factors in ICU patients (10).

Nierman et al. measured the bioavailable testosterone in the first 48 hours of ICU admissions. They found that the concentration hypotestosteronemia were below the lower limit of normal for each patients age range (11). Christeff et al., in a study on the effects of septic shock in the serum levels of estrogen and other steroidal hormones and gonadotropin in men, found that estrogen levels can be used as a prognostic factor in ICU patients (12).

Similar to the study of Ray et al., we noted that serum levels of TT-3 and TT- 4 in dead patients were significantly lower than survived patients and mortality increased with decrease of TT-3 and TT-4 blood levels (13). Rothwel et al. measured plasma levels of TT-4, TT-3, TSH and cortisol on first days of ICU admission of approximately 200 patient who did not receive dopamine and they found that thyrotropin concentration at admission can be used as a prognostic factor in ICU patients (14). Slag et al. found that T3 and cortisol levels could be used as prognostic factors in ICU patients (15). In our study, we noticed a significant correlation between TT-3 and TT-4 (on the 5th and 10th days of measurement) and mortality in traumatic ICU patients. Furthermore, risk of ICU mortality increased as plasma levels of TT-3 and TT-4 started to diminish.

We did not measure other hormones such as cortisol, DHEAS, or testosterone which could be considered as a limitation of the present study.

\section{Conclusion}

Based on findings of present study, there was a statistically significant relation between TT-3 and APACHE 4 Score and duration of hospitalization. It seems that TT3 can be utilized as a prognostic factor in the traumatic patients admitted to ICU.

\section{References}

1. Civetta JM. Prediction and definition of outcome. (1997). In: Civetta JM, Taylor RW, Kirby RR (ed) Critical care. Lippincott-Raven, Philadelphia, 24,127-147

2. Keegan MT, Gajic O, Afessa B. (2012). Comparison of APACHE III, APACHE IV, SAPS 3, and MPMOIII and Influence of Resuscitation Status on Model Performance Comparison of ICU Prognostic Models. CHEST Journ I, 142(4),851-858. 3. Plank LD, Connolly AB, Hill GL. (1998). Sequential changes in the metabolic response in severely septic patients during the first 23 days after onset of peritonitis. Ann Surg, 228,146-158.

4. Fong Y, Marono MA, Moldawer LL, et al.(1990). The acute splanchnic and peripheral tissue metabolic response to endotoxin in humans. J Clin Invest, 85,1896-1904.

5. Zaloga, G. P., Chernow, B. A. R. T., Smallridge, R. C., Zajtchuk, R. U. S. S. E. L. L., Hall-Boyer, K. A. T. H. R. Y. N., Hargraves, R. O. N. A. L. D., ... \& Burman, K. D. (1985). A longitudinal evaluation of thyroid function in critically ill surgical patients. Annals of surgery, 201(4),456.

6. KAPTEIN EM. (1986). Thyroid hormone metabolism in illness. In: HENNEMANGN, ed. Thyroid hormone metabolism New York Marcel Dekker, 25,297-333.

7. Akbari, G. Akhavan, and A. Mohammadian. (2012). Comparison of the RTS and ISS scores on prediction of survival chances in multiple trauma patients. Injury, 6,11.

8. Van der Berge G, de Zegher F, Boullion R. (1998). Acute and prolonged critical illness as differed neuroendocrine paradigms. J Clin Endocrine Metab, 83,1827-1834. 
9. Jarek MJ, Legare EJ, McDermott MT, et al. (1993). Endocrine profiles for outcome prediction from the intensive care unit. Crit Care Med, 21,543-550.

10. Wade CE, Lindberg JS, Cockrell JL, Lamiell JM, et al. (1998). Upon-admission adrenal steroidogenesis is adapted to degree of illness in intensive care unit patients. J Clin Endocrinal Metab, 67,223-227.

11. Nierman DM, Mechanick JL. (1999). Hypotesteronemia in chronically critically ill men. Crit Care Med, 27,24182421.

12. Christeff N, Carli A, Benassayag C, et al. (1992). Relationship between changes in serum estrone levels and outcome in human males with septic shock. Circ Shock, 36,249-255.

13. Ray DC, Macduff A, Drummond GB, et al. (2002). Endocrine measurements in survivors and non-survivors from critical illness. Intensive Care Med, 28,1301-1308.

14. Rothwell PM, Udwadia ZF, Lawler PG. (1993). Thyrotyropin concentration predicts outcome in critical illness. Anaesthesia, 48,373-376.

15. Slag MF, morley JE, Elson MK, et al. (1981). Hypothyroxinemia in critically ill patients as a predictor pf high mortality. JAMA, 245,34-45. 